livraisons

d'Histoire

de l'Architecture

\section{Livraisons de l'histoire de l'architecture}

13 | 2007

Architectures des établissements d'enseignement supérieur

\title{
La faculté de médecine et de pharmacie de Bordeaux par Jean-Louis Pascal (1876-1888 et 1902-1922)
}

The Faculty of Medicine and Pharmacy in Bordeaux by Jean-Louis Pascal

(1876-1888 and 1902-1922)

Die Fakultät für Medizin und Pharmazie Jean-Louis Pascals in Bordeaux

(1876-1888 und 1902-1922)

\section{Anne Richard-Bazire}

\section{OpenEdition}

\section{Journals}

Édition électronique

URL : http://journals.openedition.org/lha/414

DOI : $10.4000 /$ lha. 414

ISSN : 1960-5994

\section{Éditeur}

Association Livraisons d'histoire de l'architecture - LHA

\section{Édition imprimée}

Date de publication : 10 juin 2007

Pagination : 105-120

ISSN : 1627-4970

Référence électronique

Anne Richard-Bazire, "La faculté de médecine et de pharmacie de Bordeaux par Jean-Louis Pascal

(1876-1888 et 1902-1922) », Livraisons de l'histoire de l'architecture [En ligne], 13 | 2007, mis en ligne le 10 juin 2007, consulté le 07 mai 2019. URL : http://journals.openedition.org//ha/414 ; DOI : 10.4000/ Iha. 414

Ce document a été généré automatiquement le 7 mai 2019.

Tous droits réservés à l'Association LHA 


\title{
La faculté de médecine et de pharmacie de Bordeaux par Jean- Louis Pascal (1876-1888 et 1902-1922)
}

\author{
The Faculty of Medicine and Pharmacy in Bordeaux by Jean-Louis Pascal \\ (1876-1888 and 1902-1922) \\ Die Fakultät für Medizin und Pharmazie Jean-Louis Pascals in Bordeaux \\ (1876-1888 und 1902-1922)
}

Anne Richard-Bazire

1 La première faculté de médecine, créée à Bordeaux dès le $\mathrm{XV}^{\mathrm{e}}$ siècle, avait disparu à la Révolution pour réapparaître en 1829 sous la forme d'une école de pharmacie et de médecine ${ }^{1}$. Lors d'une délibération du 2 mars 1872, le conseil municipal de Bordeaux demanda la transformation de son école préparatoire en faculté de médecine et de pharmacie. « Depuis la guerre de 1870, il y avait en France un véritable engouement pour toutes les institutions allemandes. L'Allemagne possédait de nombreux centres universitaires, la France voulait en créer également, et Bordeaux se fût cru déshérité s'il n'eût été doté d'une université complète $»^{2}$. La loi du 8 septembre 1874 transforma l'école bordelaise en faculté mixte de médecine et de pharmacie ${ }^{3}$. Les locaux de l'ancienne école de médecine de la rue Lalande, aménagée en 1852 par l'architecte de la ville, Charles Burguet, autour de l'ancien amphithéâtre Saint-Cosme ${ }^{4}$, se révélèrent vite insuffisants. On appropria alors, à grands frais, les locaux de la caserne Saint-Raphaël, pour loger les étudiants provisoirement. Le lendemain du jour où le conseil municipal votait le budget définitif de la faculté de médecine, l'arrêté du 22 juillet 1878 modifiait la circonscription de la faculté lui enlevant quatre départements : les Landes, les Basses-Pyrénées, le Lot-etGaronne et la Dordogne, ce qui diminuait ses recettes de quatorze mille francs ${ }^{5}$. Or la ville avait déjà fait des dépenses énormes ${ }^{6}$, mais c'était avec l'idée que la nouvelle faculté sans rivale dans le sud-ouest, ne tarderait pas à recevoir un nombre d'étudiants bientôt 
suffisant ${ }^{7}$ pour couvrir ses dépenses annuelles. La création d'une faculté de médecine à Toulouse, en altérant profondément le pacte conclu avec l'État, dégageait la ville. Dès lors, il fut décidé de surseoir à la construction d'une nouvelle faculté qui avait été prévue sur le terrain de l'ancien hôpital des Incurables à Saint-Julien, jusqu'à ce que l'État ait accordé à la ville une compensation pour le préjudice que lui causait la création d'une faculté rivale. Sur ces entrefaites, Jules Ferry vint à Bordeaux et il proposa à la ville une combinaison qui servit de base à la nouvelle convention présentée par Louis Liard $(1846-1917)^{8}$, adjoint au maire à l'instruction publique, au conseil municipal, le 18 novembre $1879^{9}$.

\section{Le concours}

2 Un concours est ouvert le 4 avril 1876 pour créer un nouveau bâtiment devant couvrir les terrains de l'ancien hôpital des Incurables, bordés à l'ouest par la place d'Aquitaine (actuelle place de la Victoire) et la rue Candale, au nord par la rue Bigot (Paul Broca), au sud par la rue des Incurables (Élie Gintrac), à l'est par la rue Leyteire.

3 La volonté du maître d'ouvrage, l'État, est de construire un bâtiment fonctionnel, moderne, et utilisant autant que possible les matériaux en usage à Bordeaux afin de réduire les dépenses : une volonté de rationaliser la construction par souci d'économie ${ }^{10}$.

Ce que nous devons chercher aujourd'hui, ce sont des constructions conçues de telle sorte que chacun des locaux soit adapté à sa destination spéciale et placée en son lieu voulu. J'ajoute que notre préoccupation commune doit être beaucoup moins de construire un monument, ce qui comporterait des dépenses d'ornementation toujours très onéreuses, que d'édifier une grande école pourvue de toutes les ressources indispensables [...] ce qu'il nous faut avant tout, c'est la solidité, des étendues proportionnées aux besoins, des communications faciles entre les différentes parties des bâtiments, de l'air et de la lumière; c'est-à-dire les satisfactions essentielles réclamées au point de vue du travail, de l'hygiène et de la discipline ${ }^{11}$.

4 Le programme ${ }^{12}$, qui insiste sur l'aération des locaux, leur chauffage ${ }^{13}$ et la distribution abondante de la lumière dans les laboratoires ${ }^{14}$, énumère les différentes sections qui devront s'organiser sur une superficie de $9426 \mathrm{~m}^{2}$ : la section d'anatomie, de physiologie, de physique, de chimie, d'histoire naturelle, de pharmacie et de toxicologie, d'anatomie pathologique et d'histologie.

Outre les logements de fonction, le bâtiment devra accueillir un musée d'anatomie, une bibliothèque, un grand amphithéâtre et deux plus restreints, de nombreux laboratoires et salles d'étude, un jardin botanique de $2500 \mathrm{~m}^{2}$ comprenant une serre chaude et une serre froide. De plus, si les laboratoires devaient être en communication directe avec les salles de cours ${ }^{15}$, les salles de dissection devaient être isolées ${ }^{16}$. Le texte du concours demandait que la séparation entre les services de médecine et de pharmacie ne fût pas totale. Le montant de la dépense était un des éléments décisifs du choix à faire entre les projets. Les architectes ne signeraient pas leurs projets, mais y apposeraient une épigraphe ou une devise ${ }^{17}$. La date de clôture du concours était le 15 septembre 1876. Une exposition publique de huit jours ${ }^{18}$ précédait l'envoi à Paris, lieu de jugement du concours ${ }^{19}$.

Cinquante-trois projets sont présentés au jury. Dix-huit d'entre eux sont retenus, après une élimination de trente-cinq projets jugés insuffisants. Sept projets sont ensuite conservés, mais ils dépassent tous l'enveloppe budgétaire prévue. 
Il n'y a pas de premier prix - on accorde un deuxième prix à Alfred Leclerc (1843-1915) -, le troisième prix ex æquo est attribué à Théodore Dauphin (1849-1917) et Jean-Louis Pascal (1837-1920) ${ }^{20}$, le cinquième à Ferdinand Dutert (1845-1906), le sixième à Albert Ballu (1849-1939) (fils de Théodore Ballu). La forme trapézoïdale et l'exiguïté du terrain entraînent des plans assez semblables, orientant l'édifice est-ouest et proposant pour la façade principale un développement sur le petit côté, celui donnant sur la place d'Aquitaine ${ }^{21}$.

Jean-Louis Pascal remporta le concours, car l'estimation du coût de son projet restait dans les limites fixées par le programme (un million cinq cent mille francs) ; il dut cependant rejeter le jardin à l'arrière de l'édifice ${ }^{22}$, opérant une sorte de " glissement ${ }^{23}$ de son plan vers l'avant, tout en conservant un parvis pour les étudiants, et remplaça, pour des raisons économiques ${ }^{24}$, le grand amphithéâtre par une cour d'honneur (ill. 1).

III. 1 : Plans de la faculté de médecine et de pharmacie de Jean-Louis Pascal, place de la Victoire à Bordeaux.

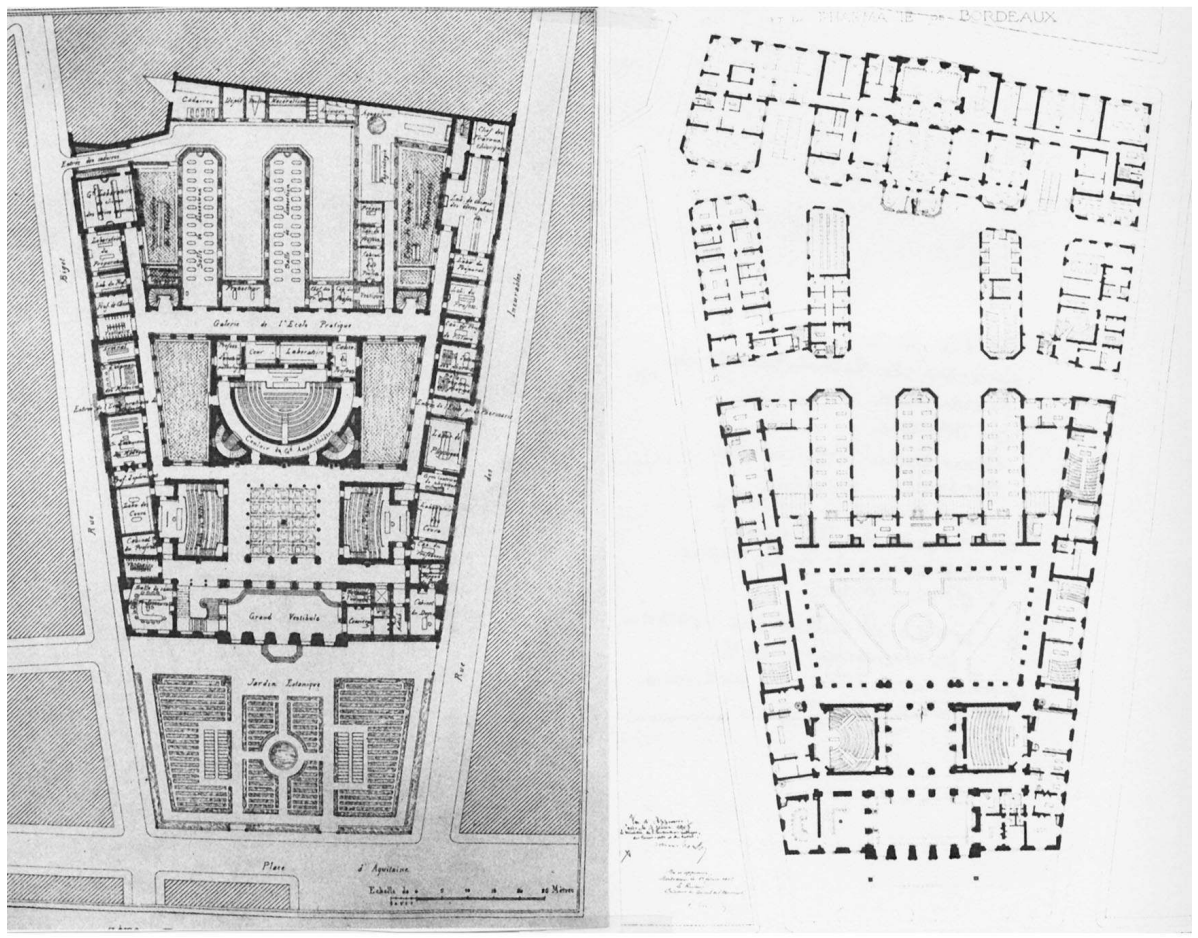

Claude Laroche, Revue archéologique de Bordeaux, tome LXXXIII, année 1992. Projet de Pascal, 3e prix ex æquo, plan, 1876, (avec implantation antérieure du jardin), p. 145

Projet d'extension, plan (avec la pharmacie), 1902, p. 148

L'architecte, ancien élève de Jacques Gilbert et de Charles Questel, et disciple fidèle de Charles Garnier, est un pur produit de l'École des beaux-arts. Grand prix de Rome en 1866, il a œuvré cinq ans à l'agence de l'Opéra de Paris, puis au Louvre sous la direction d'Henri Lefuel. Lorsque la construction de la faculté lui échoit, c'est un architecte reconnu, en charge de la Bibliothèque nationale depuis 1875, architecte diocésain de Valence et bientôt d'Avignon. Il dirige d'autre part, depuis 1872, le prestigieux atelier de ses anciens maitres, rue Mazarine.

11 Les travaux de la faculté de Bordeaux commencent le 12 mai 1880. 


\section{La réalisation}

\section{Un bâtiment « rationnel »}

\section{La fonctionnalité des espaces}

Chacun sait que la forme ou le lieu d'un atelier ou d'un laboratoire n'ont pas grand effet pour obtenir des résultats, tandis que pour le but que doit atteindre une École, c'est-à-dire pour l'élévation de la moyenne, les facilités, les commodités d'installation, les améliorations, le groupement en un mot sont des facteurs puissants du succès ${ }^{25}$.

Le plan de la faculté de médecine s'organise ainsi : on entre par trois grandes portes dans un vestibule dont le dallage en mosaïque ${ }^{26}$ dessine au sol la devise choisie par Pascal comme pseudonyme au concours : «Pro Scientia Urbe et Patria ». Il a été réalisé en 1888 par le mosaïste vénitien Jean-Dominique Facchina (1826-1903) qui avait travaillé avec Pascal au monument à Regnault dans la cour du mûrier à l'École des beaux-arts et avec Garnier à l'opéra de Paris. Vient ensuite une galerie transversale puis un atrium, doublé $\mathrm{du}$ portique de la cour d'honneur où s'ouvrent les hautes portes des laboratoires de dissection (ill. 2).

\section{2 : Faculté de médecine et de pharmacie de Bordeaux de Jean-Louis Pascal}

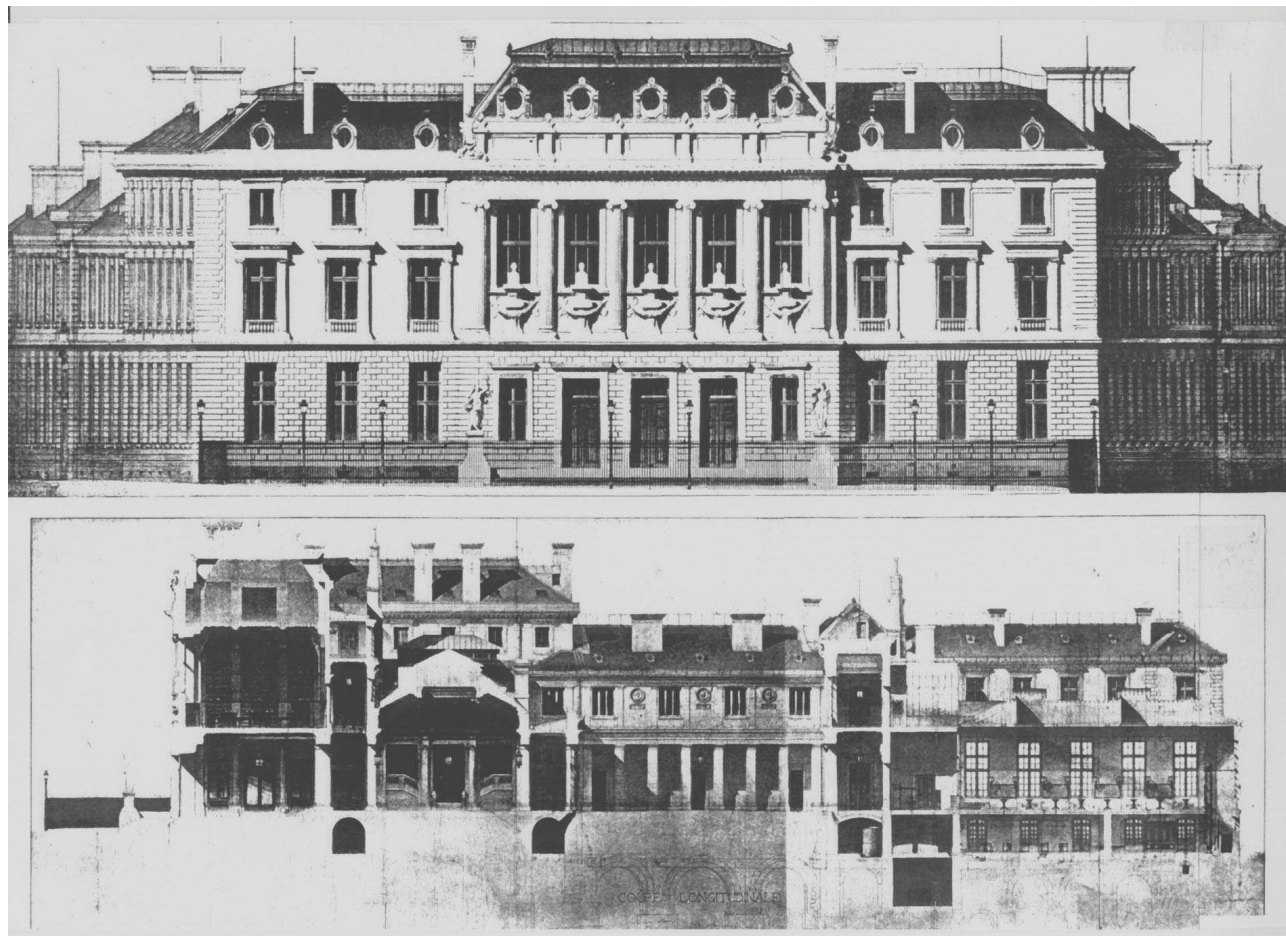

Façade et coupe

Jean-Louis Pascal, L'Architecture, 1895, p. I-VI.

L'atrium (ill. 3), carré, mosaïqué, est scandé de colonnes ioniques engagées, à fûts lisses, supportant un entablement à ressauts. 
III. 3 : Atrium de la faculté de médecine et de pharmacie de Jean-Louis Pascal à Bordeaux

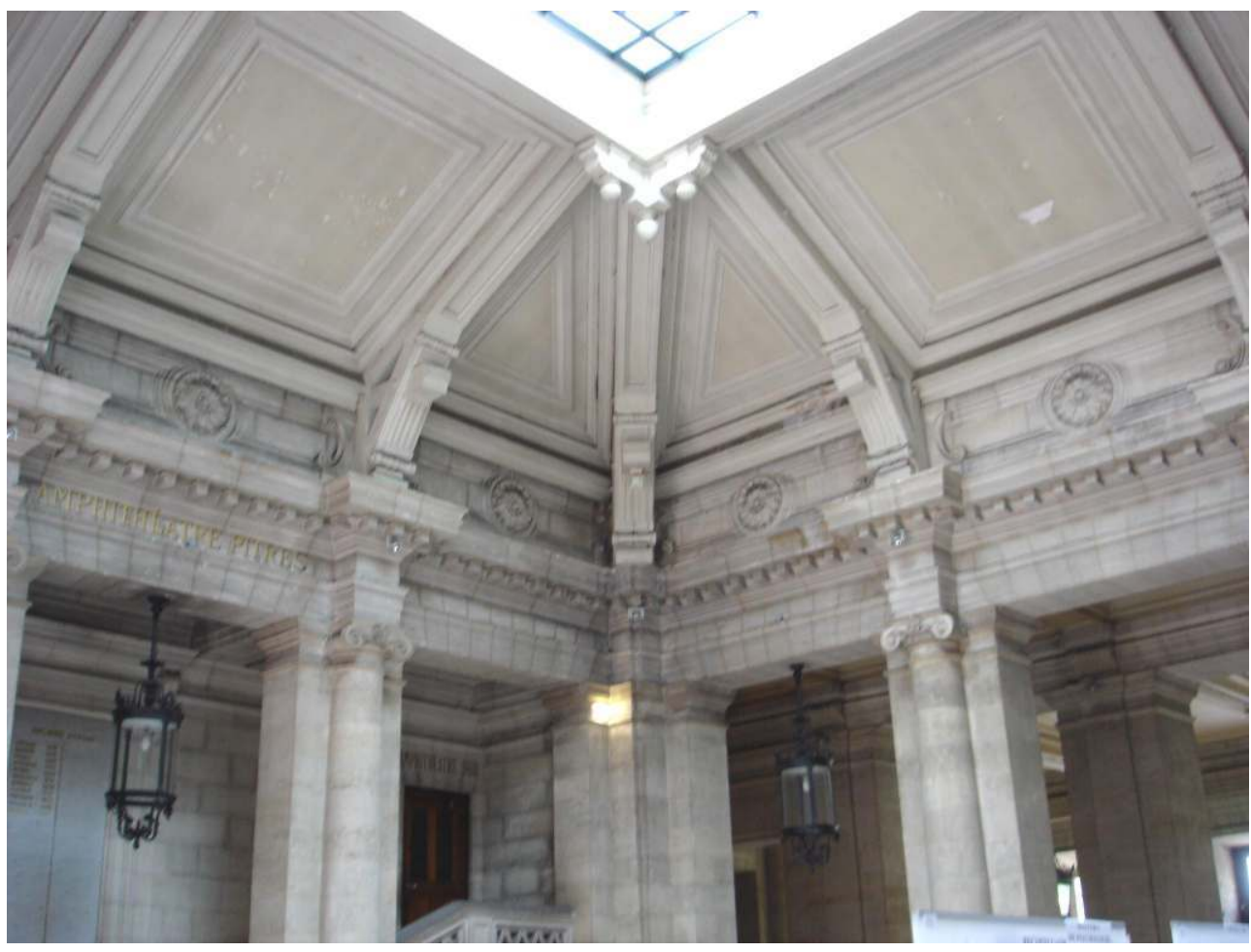

Cl. A. Richard-Bazire

14 Il est couronné d'un comble en charpente apparente portant un toit en pavillon à terrasse faîtière vitrée, et amplifié des deux escaliers doubles en pierre, menant aux deux amphithéâtres principaux de deux cent vingt places auxquels s'ajoute un laboratoire.

La pente est plus raide dans l'amphithéâtre d'anatomie pour permettre une bonne vision de la table de dissection. Chaque élève a un petit dossier sur lequel s'appuie une tablette pour le voisin du niveau supérieur ${ }^{27}$ (ill. 4). 
III. 4 : Amphithéâtre Gintrac de la faculté de médecine et de pharmacie de Jean-Louis Pascal à Bordeaux

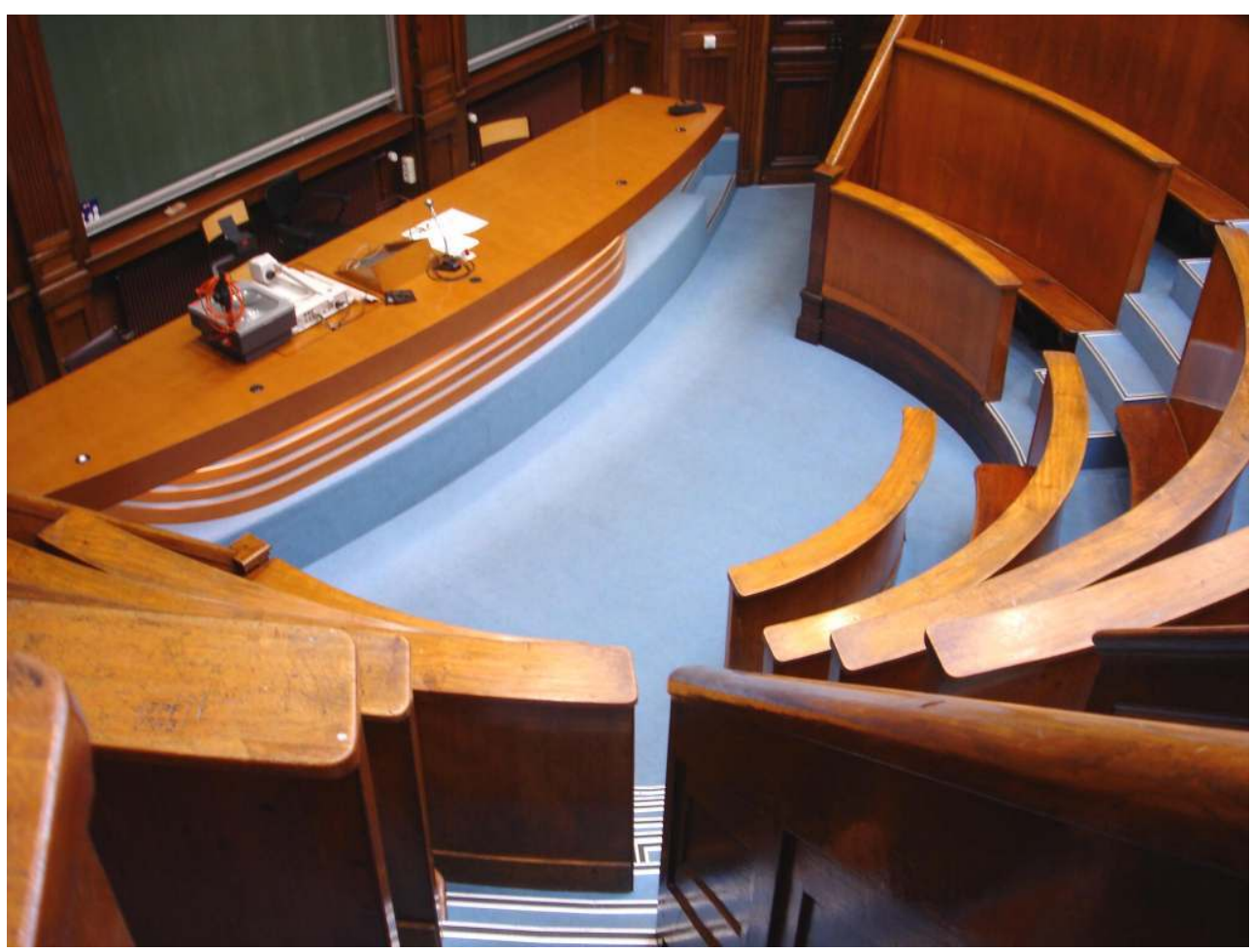

Cl. A. Richard-Bazire

16 L'éclairage du soir se fait au gaz et le chauffage, par des calorifères placés en dessous des locaux.

17 La cour d'honneur en trapèze est entourée de portiques ouverts au rez-de-chaussée, de galeries vitrées au premier étage. Un ordre ionique, à coussinet convexe ${ }^{28}$ marque les points forts du portique du rez-de-chaussée, surmonté sur trois côtés d'un étage où alternent baies et trumeaux ornés des médaillons de personnages scientifiques du sudouest (ill. 5). 


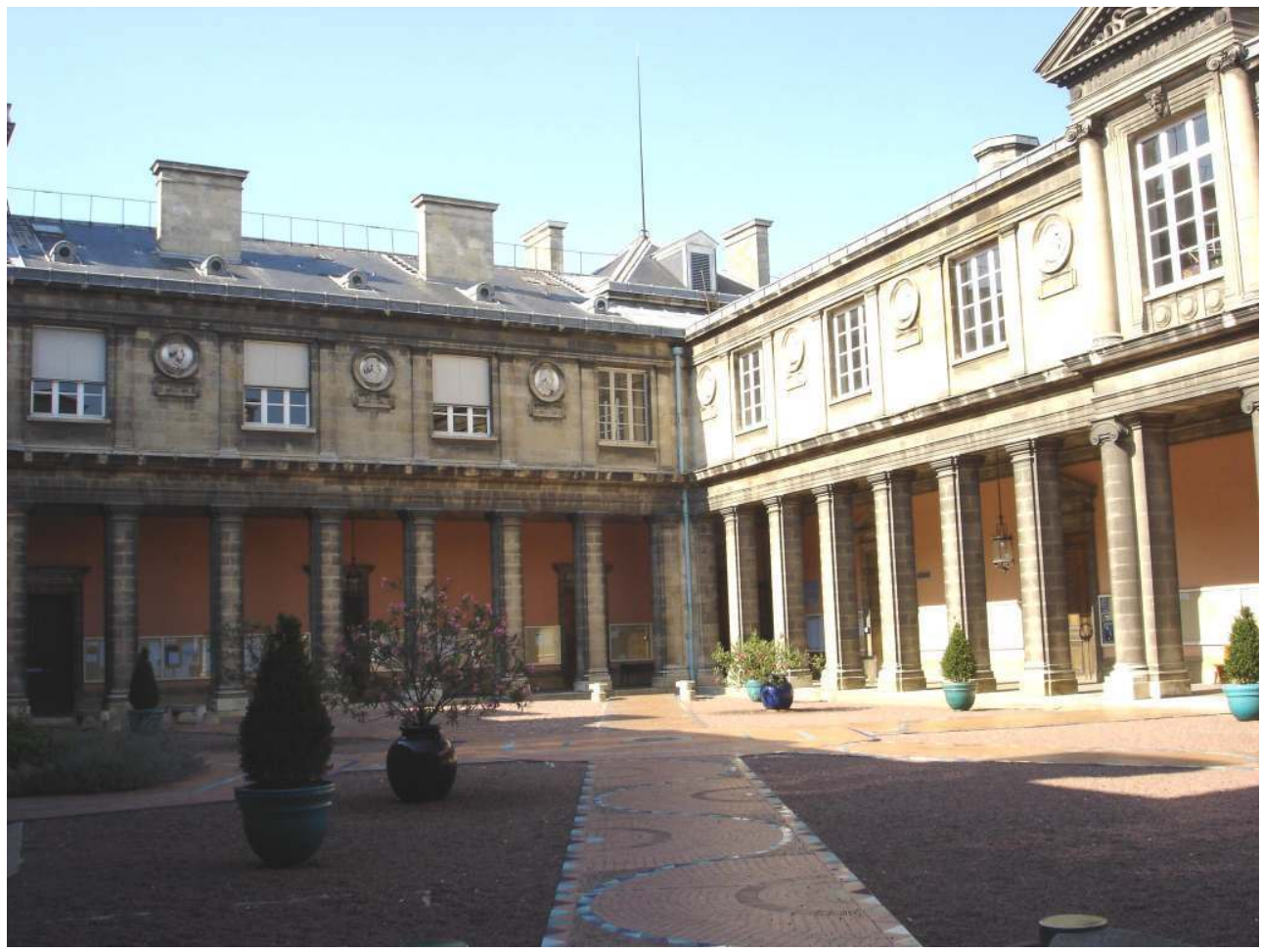

Cl. A. Richard-Bazire

18 Les galeries parallèles à la rue Broca et Gintrac desservent les laboratoires et les salles d'examen avec chacune un amphithéâtre de soixante-douze places. Sur la rue Broca se situe le laboratoire de médecine expérimentale. Au premier étage se trouvent les deux instituts d'histologie et d'anatomie pathologique. Sur la rue Gintrac est situé le laboratoire de pathologie générale et de physiologie et au premier étage se trouvent l'histologie et la médecine légale.

Partout des tables à microscope, des stores transparents et opaques, des installations de gaz et d'eau, des lavabos, des appareils de chauffe.

20 Au milieu de la galerie de communication du premier étage au fond de la cour, se trouve un cabinet de photo; sur la partie antérieure de l'édifice, côté Broca et Gintrac, se trouvent les musées ${ }^{29}$ et un local pour le conservateur.

\section{Des matériaux nouveaux}

Jean-Louis Pascal n'hésite pas, dès qu'il le peut, à employer des matériaux nouveaux ou des techniques nouvelles pour améliorer la construction de son bâtiment :

L'exposition des arts décoratifs, ouverte en ce moment à Paris, m'a donné l'occasion de voir les résultats de l'application d'un procédé analogue à celui de la silicatisation mais préconisé par ceux qui l'exploitent comme de beaucoup supérieur à ce dernier, dont les inconvénients sont nombreux. Il s'agit du durcissement des pierres tendres par une opération chimique qui n'en altèrerait pas la couleur et l'aspect [...] J'aimerais à en faire un essai sur une échelle assez importante et l'occasion m'en est offerte par la nécessité de ravaler les souches des cheminées de la faculté de médecine avant qu'on pose la couverture [...] un essai de fluatation [sic], c'est le nom qu'on donne au procédé de MM. Faure et Kessler de ClermontFerrand. J'ajouterai que le durcissement pouvant s'appliquer aux enduits, crépis et ciments, 
l'épreuve à faire pourrait avoir de nombreuses applications ultérieures dans notre monument et ailleurs à Bordeaux ${ }^{30}$.

Jean-Louis Pascal va innover pour la construction des escaliers principaux de la faculté qui étaient prévus en pierre de Montbron. L'usage ayant démontré les inconvénients des escaliers en saillie avec simple encastrement dans le mur, Pascal pensa qu'il était prudent et économique de substituer un mode de construction mixte, fer et pierre, à celui prévu. Pour éviter le déversement d'escaliers à grande portée, tel l'escalier d'honneur de deux mètres quatre-vingt-dix de large, Pascal préconise un système qui consiste à établir en fer l'ossature de l'escalier : marches, contremarches, paliers et limons, à hourdir le tout en béton de mâchefer et à recouvrir simplement le dessus, de marches ou de plaquettes en pierre très dure ${ }^{31}$. Pascal proposait d'en confier l'exécution à la maison Pozzi et Gauthier ${ }^{32}$ , de Paris, qui se serait chargée de la partie métallique, de la fourniture et de la mise en place des marches en pierre de Comblanchien, pierre utilisée pour les plaquettes et le dallage des paliers des escaliers ${ }^{33}$. L'architecte demanda son avis à Honoré Daumet (architecte du Palais de Justice de Paris) qui attesta de la grande compétence de cette maison $^{34}$. Pascal réussit à imposer cette solution mixte, fer et pierre, pour tous les escaliers principaux de la faculté, malgré la dépense supplémentaire que cela entraînait ${ }^{35}$. L'escalier principal présente un limon de forme à la française en fer et fonte et une rampe en fer forgé ; les escaliers de l'École pratique sont de forme à l'anglaise ${ }^{36}$.

Le centre du plafond du grand escalier est orné d'un grand cartouche avec guirlandes et cornets d'abondance. C'est un ornement en staff réalisé par le sculpteur Hamel sur des dessins fournis par Pascal ${ }^{37}$. Pour des questions de sécurité, Jean-Louis Pascal va également décider de construire en fer toutes les poutres maîtresses du plancher haut du rez-de-chaussée, ne conservant en bois que le remplissage du solivage ${ }^{38}$.

\section{Un bâtiment type « Beaux-arts »}

Le 28 avril 1888, la faculté de médecine est inaugurée (ill. 6). 
III. 6 : Façade de la faculté de médecine et de pharmacie de Jean-Louis Pascal à Bordeaux

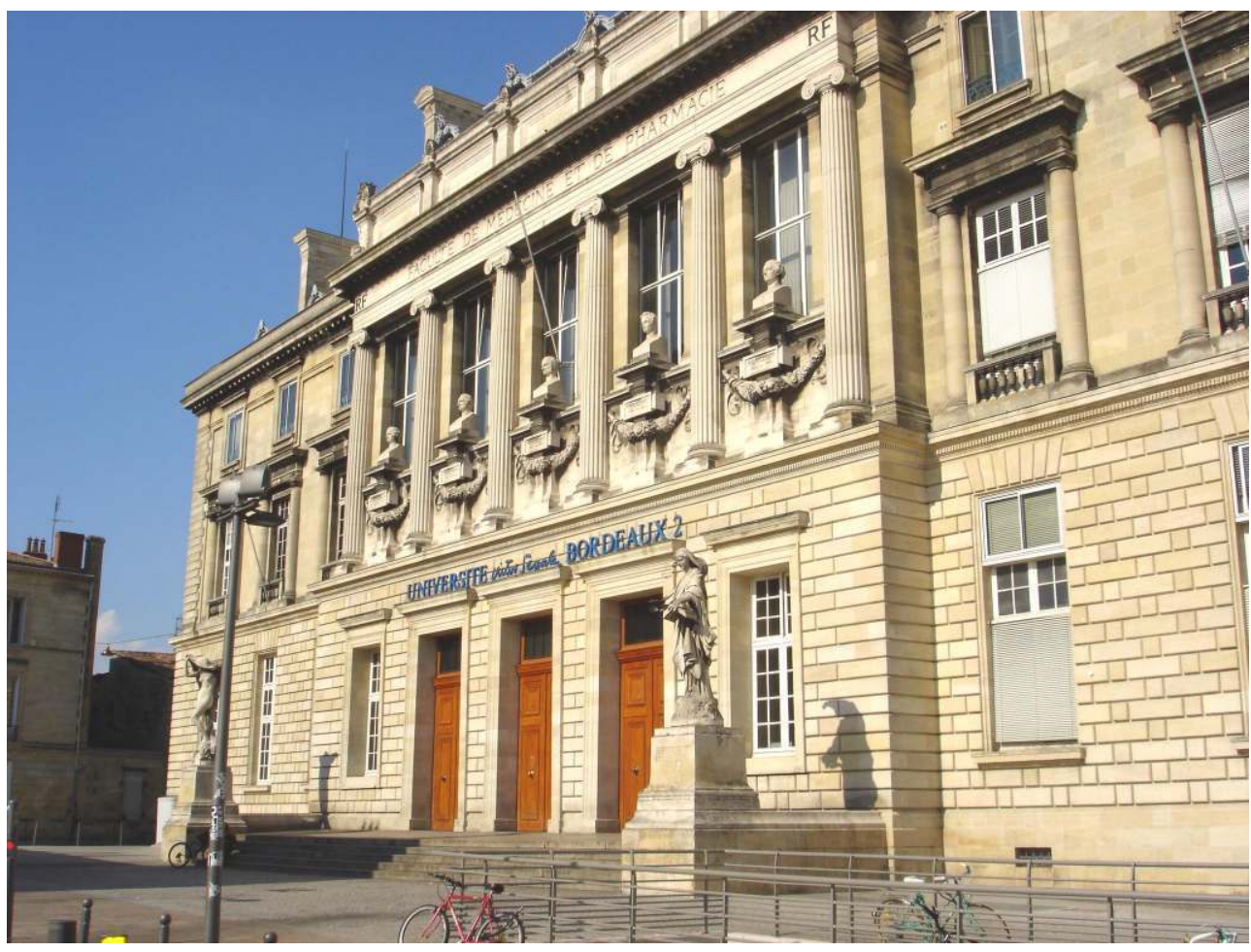

CL. A. RICHARD-BAZIRE

Dès $1884^{39}$, Jean-Louis Pascal avait décidé d'ériger deux statues monumentales ${ }^{40}$ en marbre devant la façade : «Les sujets sont sur ma proposition : la Nature et la Science, au seuil des études médicales " ${ }^{41}$. Jean-Louis Pascal souhaitait que la "Nature " fût attribuée à Louis-Ernest Barrias (1841-1905) et la « Science » à Charles-Marie Degeorge (1837-1888) ${ }^{42}$. Ce fut l'occasion d'une première passe d'armes entre l'État et la ville : « Est-ce l'État qui a décidé que les deux statues devant orner la façade principale seraient données à des sculpteurs étrangers? $\gg^{43}$ se demandait un membre du conseil municipal. Jean-Louis Pascal maintint sa volonté d'avoir deux artistes de premier ordre: «J'ai fait très bon marché du choix modeste des artistes désignés pour les médaillons de la cour, mais je voudrais que les deux statues de la façade fussent des œuvres de maître, et je vous supplie avec insistance de vouloir bien vous mettre d'accord avec moi $»^{44}$, écrivit-il au directeur des beaux-arts. Charles Degeorge étant décédé (1888), la Science fut sculptée par PierreJules Cavelier (1814-1896). Elle est « drapée de longs plis dans l'attitude de la réflexion et de l'attention ». Barrias sculptera la « Nature se dévoilant à la Science ", " sous les traits d'une femme soulevant de ses deux bras, d'un geste lent et comme à regret, les voiles dont elle était enveloppée ». Elle sera présentée d'abord au salon des Champs-Élysées : «La Nature de Barrias brille par la beauté, la pureté des formes ; l'expression générale est puissante [...] La Science de Cavelier, tenant un livre ouvert, est d'un aspect peu plus sévère ; l'expression est plus contenue ; mais l'inspiration est haute. ${ }^{45}$

Les 26 et 27 novembre 1893, les deux statues sont mises en place ${ }^{46}$ après avoir été acheminées par la Compagnie des chemins de fer Paris-Orléans. L'État prenait à sa charge la moitié de la dépense, plus la fourniture des blocs de marbre. La Ville de Bordeaux payait l'autre moitié. La même répartition était adoptée pour les bustes de marbre de la façade et les médaillons de la décoration intérieure ${ }^{47}$. Jean-Louis Pascal souhaitait ajouter 
d'autres figures allégoriques, dans l'escalier par exemple, et quelques œuvres picturales pour décorer les amphithéâtres.

Dès 1884, Pascal décide aussi de représenter en silhouette dans les grandes baies de la façade, cinq bustes ${ }^{48}$, posés sur des gaines saillantes, devant lesquelles passent des guirlandes de fruits et de feuillages. Ils représentent la botanique : Bernard de Jussieu ${ }^{49}$ (1699-1777), la médecine: René Laënnec ${ }^{50}$ (1781-1826), l'anatomie: Xavier Bichat ${ }^{51}$ (1771-1802), la chirurgie : Guillaume Dupuytren ${ }^{52}$ (1777-1835), et la chimie: AntoineLaurent de Lavoisier $^{53}$ (1743-1794). Ces bustes marquent l'espace privilégié de la bibliothèque; ils sont la «signature » du bâtiment, «caractérisant par le choix des personnes représentées: soit les sciences principales qui constituent l'étude de la médecine, par l'effigie des hommes les plus éminents dans ces spécialités, soit l'histoire même de l'art de guérir par la représentation de ses personnalités les plus marquantes depuis l'Antiquité jusqu'à nos jours. Ce choix ${ }^{54}$, qui échappe à ma compétence [celle de Pascal], sera fait par le doyen de la faculté de médecine, le Recteur et l'administration municipale ». C'est le doyen qui choisit de faire figurer des grands noms de la Science et non des gloires locales, à qui il réservait l'intitulé des rues entourant la faculté : « Il m’a semblé que, envisagée de la sorte, la question scientifique se rétrécissait singulièrement, et qu'en mettant en avant des illustrations locales, on risquait de diminuer la grandeur des figures sous le patronage desquelles nous cherchons à placer notre faculté $»^{55}$. JeanLouis Pascal se montra en revanche très ferme quant au choix des sculpteurs :

Personnellement, j'ai le plus vif désir qu'aucune de ces œuvres ne soit inférieure, et c'est la raison pour laquelle je souhaiterais que l'État voulût bien approuver la répartition que j'ai faite de la sculpture du monument, en laissant à la désignation de votre administration des beaux-arts toute la sculpture de la façade principale, tandis que la ville de Bordeaux répartira, peut-être entre des artistes locaux, les nombreux médaillons de professeurs, de savants ou de médecins illustres, qui sont prévus pour décorer le premier étage de la cour, sans parler des figures allégoriques, et de la sculpture purement décorative où la représentation de la figure humaine ne jouerait qu'un rôle accessoir ${ }^{56}$.

En fait, Pascal sera aussi très attentif à la réalisation de la sculpture des quatorze effigies en médaillons qui devaient orner la cour d'honneur ${ }^{57}$ :

J'ai successivement visité les ateliers de MM. Basset, Maximilien Bourgeois et Leclaire, statuaires chargés de trois médaillons pour la faculté de médecine de Bordeaux. Les artistes ont tenu compte de toutes les prescriptions détaillées que je leur avais fournies pour que leurs œuvres concourussent à l'ensemble harmonieux de la cour qu'ils sont chargés de décorer [...] J'attache le plus grand prix à avoir des œuvres de premier ordre pour compléter une façade à laquelle j'ai donné tous mes soins ${ }^{58}$.

Le conseil de la faculté en a choisi les sujets ${ }^{59}$, mais c'est le ministère qui choisit les artistes, la municipalité essayant tout de même de placer quelques artistes locaux ${ }^{60}$. Quant à la sculpture décorative, Jean-Louis Pascal imposera que les modèles en fussent faits à Paris par l'entrepreneur parisien Hamel dont il appréciait beaucoup le travail. Jean-Louis Pascal jugeait les artistes bordelais comme des « exécutants habiles, mais trop faciles à contenter ${ }^{61}$. Pourtant, pour avoir les prix les plus bas, il met en concurrence artistes parisiens et bordelais, se réservant le choix final. C'est Hamel qui est choisi car, bien que parisien, il est le moins cher ${ }^{62}$.

Je souhaiterais, tenant plus que je ne saurais dire au style, au caractère, que l'architecture très simple de mon monument doit présenter, que les modèles de ces sculptures fussent faits ici, sous mon contrôle personnel et fréquent, peut-être même par les sculpteurs que l'habitude de travailler sous ma direction rend plus aptes à bien interpréter ce qui doit s'associer avec l'esprit même de mon architecture ${ }^{63}$. 
ace de la faculté étant exposée à l'ouest, aux vents pluvieux de l'océan, Jean-Louis Pascal demandait que les bustes de la façade fussent réalisés en marbre, ou tout au moins dans une pierre très résistante. Et il proposait de choisir les sculpteurs Delaplanche, Mercié, Hiolle, Allar ${ }^{64}$. Le directeur des beaux-arts conditionna alors sa participation à la moitié des frais, en échange du choix par l'architecte et la municipalité, de cinq sculpteurs dans une liste fournie par lui « d'artistes jeunes, connus et distingués » ${ }^{65}$. JeanLouis Pascal y choisit les Parisiens Gustave Michel (Bichat), Camille Lefebvre (Laënnec) et Charles Ghautier (Lavoisier), « trois hommes de talent qui, je l'espère, nous donneront de beaux motifs décoratifs $»^{66}$ et la municipalité choisit les Bordelais Edmond Prévot (Dupuytren) et Pierre Granet (Jussieu).

31 Pascal s'était renseigné sur Prévot: «Un homme fortement recommandé par une personnalité autrefois bordelaise [Liard] qu'il est de l'intérêt de tous de contenter. Les figures ${ }^{67}$ que je connais de cet artiste, à l'entrée de la cour de votre hôtel de ville, me font croire qu'il ne serait pas trop indigne de figurer à côté des sculpteurs désignés par l'État » 68 .

Le décor de la salle des actes $^{69}$ (ill. 7) est confié aux deux décorateurs du théâtre de Bordeaux, Artus et Lauriol.

\section{7 : Salle des Actes de la faculté de médecine et de pharmacie de Jean-Louis Pascal à Bordeaux.}

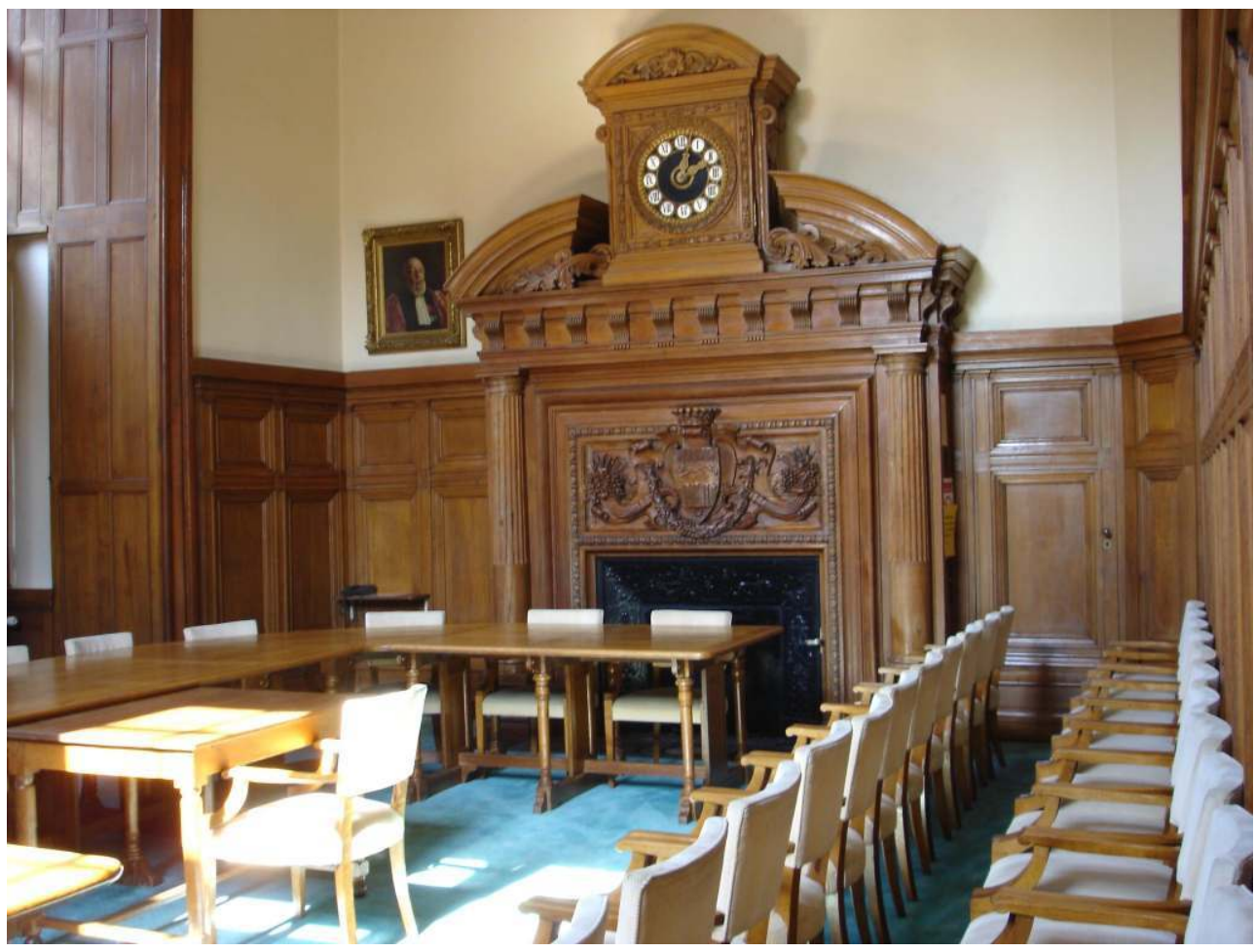

Cl. A. Richard-Bazire

Elle est recouverte sur tout son pourtour, et sur une hauteur de deux mètres trente, de lambris de chêne à moulures et à grands panneaux. Une cheminée ${ }^{70}$ en bois de chêne en constitue le point d'orgue. De son fronton interrompu, s'échappe une horloge, elle-même surmontée d'un petit fronton semi-circulaire orné d'une rosace et de motifs végétaux, et accotée de deux consoles terminées par de grands culots d'acanthe. Le manteau est orné d'un grand motif avec cartouche aux armes de la Ville, orné de cornes d'abondance, 
guirlandes de laurier et couronne comtale $e^{71}$. Le mobilier est à l'époque constitué d'une grande table de chêne ciré en forme de fer à cheval, recouverte d'un tapis en drap vert frangé. Douze fauteuils et un grand bureau avec estrade, pour le président, complètent l'aménagement de cette salle ${ }^{72}$. Des garnitures de croisées en velours de lin, doublé en andrinople avec gros et larges galons renaissance sur le devant et dans le bas, et franges en passementerie sur le bord avec lambrequin habillaient les fenêtres ${ }^{73}$. « La consultation à l'hôpital de Saint-Cloud $\aleph^{74}$ tableau peint par Édouard Dantan et acquis par l'État au Salon de 1888, "l'un des tableaux les plus remarqués du Salon de cette année $»^{75}$ est destiné au cabinet du Doyen. La ville, elle, finance pour moitié ${ }^{76}$ « La remise solennelle de la faculté à M. le Président de la République » du même artiste ${ }^{77}$, tableau de 1889 (ill. 8), destiné à la décoration de la salle des actes ${ }^{78}$.

III. 8 : Tableau d'Édouard Dantan (1848-1897) : « La remise solennelle de la faculté à M. le Président de la République»

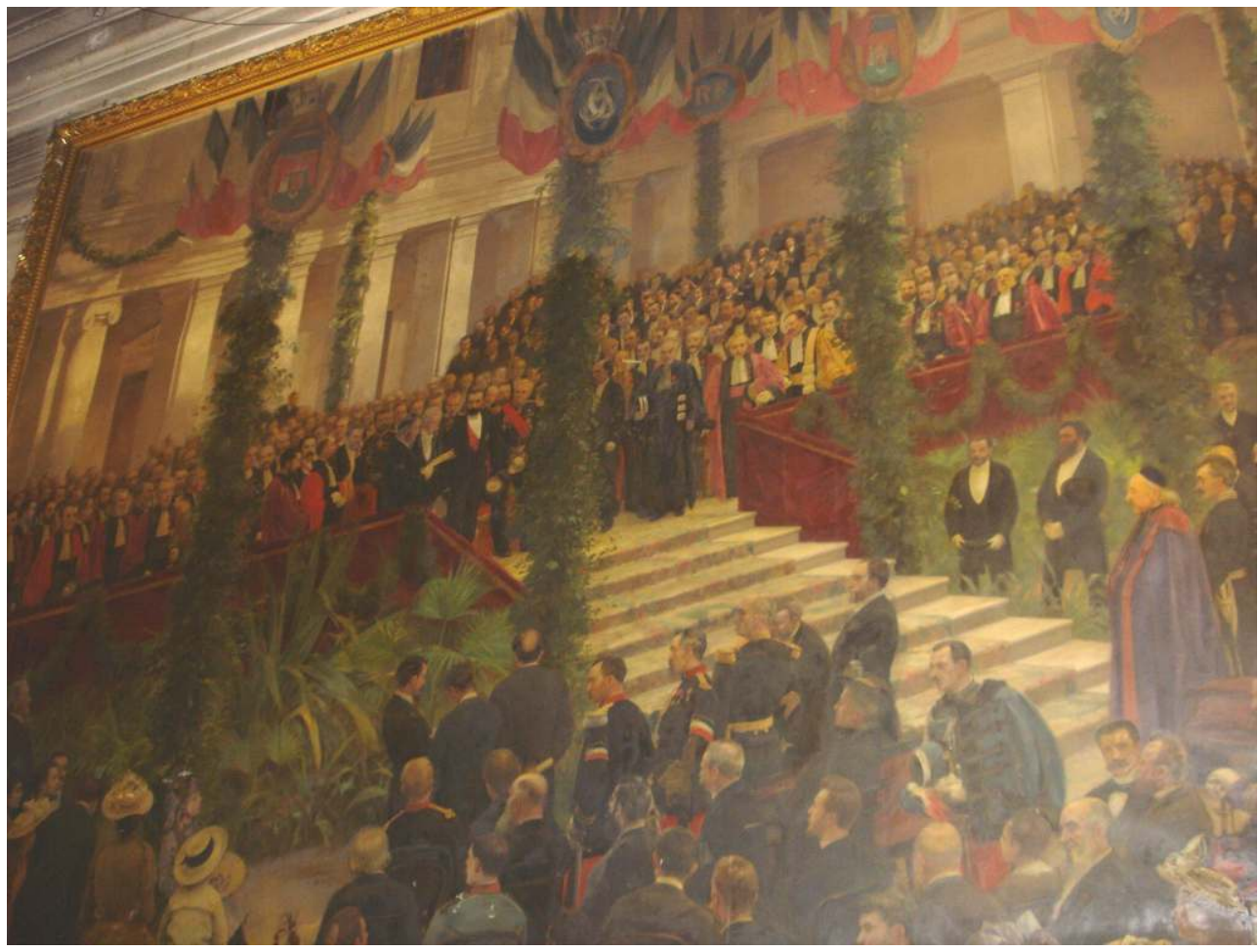

1889, mur N.E. de la galerie située à l'arrière du vestibule d'entrée de la faculté de médecine et de pharmacie de Jean-Louis Pascal à Bordeaux

Cl. A. Richard-Bazire.

ouis Pascal s'était lié d'amitié avec Édouard Gerspach, directeur de la manufacture des Gobelins, avec qui il avait été en relation pour les tapisseries de la Bibliothèque nationale ${ }^{79}$, et celui-ci lui offrit d'exécuter deux panneaux symbolisant la médecine et la pharmacie ${ }^{80}$ pour le grand escalier de la faculté, à charge pour la municipalité de payer ${ }^{81}$ les cartons exécutés par Galland, à la demande de Pascal ${ }^{82}$. Elles sont mises en place le 24 mai 1892.

Pascal n'avait pas oublié l'idée d'une faculté mixte de médecine et de pharmacie. La partie arrière de l'îlot qui se prolonge par des maisons jusqu'à la rue Leyteire sera achetée à cet effet par la ville en 1899. La dépense des opérations était supportée à moitié par la Ville, 
et à moitié par l'État et l'Université. La subvention de l'État (1 071000 francs) ne devait être en aucun cas augmentée ${ }^{83}$.

Pascal signe les plans définitifs de cette extension le 28 janvier 1902. Les travaux qui ne commencent qu'en 1908, se poursuivent jusqu'en 1914. Ils reprennent après la guerre, et le décès de Pascal, et s'achèveront en 1922, sous la direction de Paul Mialhe comme architecte suppléant au début du chantier, puis sous celle d'Ernest Lacombe. Á la suite de réformes pédagogiques, certains locaux seront transformés en laboratoires.

\section{NOTES}

1. Claude Laroche, Dominique Dussol, «Les facultés de Bordeaux", La Sorbonne et sa reconstruction, sous la direction de Philippe Rivé, Paris, la Manufacture, 1987, p. 201-222. Claude Laroche, «Pro Scientia Urbe et Patria : l'architecture de la faculté de médecine et de pharmacie de Bordeaux, 1876-1888 et 1902-1922", Revue archéologique de Bordeaux, tome LXXXIII, année 1992, p. 137-173. Marc Saboya et Robert Coustet, Bordeaux, le temps de l'histoire. Architecture et urbanisme au XIXe siècle (1800-1914), Bordeaux, Éd. Mollat, 1999, 271 p.

2. Arch. mun. Bordeaux, 6872 M 25, A. Gambier, «La faculté de médecine », La Victoire de la démocratie, mardi 18 novembre $1879,10^{\mathrm{e}}$ année, $\mathrm{n}^{\circ} 1637$.

3. Arch. mun. Bordeaux, $6872 \mathrm{M} 27$. Conseil municipal. Installation d'une faculté mixte de médecine et de pharmacie. Rapport présenté par Albert Brandenburg au nom de la commission spéciale de l'emprunt, séance du 25 février 1876.

4. Construit vers 1753 pour les maîtres chirurgiens de la ville.

5. Lettre 7 décembre 1878 du maire de Bordeaux Albert Brandenbourg au ministère, citée par A. Gambier, «La faculté de médecine », La Victoire de la démocratie, mardi 18 novembre 1879, $10^{\mathrm{e}}$ année, $n^{\circ} 1637$, Arch. mun. Bordeaux, 6872 M 25.

6. Au départ, il s'agissait seulement de modifier l'ancienne école de la rue Lalande pour 330000 francs. Le directeur de l'école préparatoire est Gintrac, avec 150000 francs de dépenses annuelles. Puis le ministre de l'instruction publique déclara ces offres insuffisantes et le conseil municipal vota des sommes s'élevant à plus de trois millions de francs avec un budget annuel de 261000 francs. Arch. mun. Bordeaux, 6872 M 25A. Gambier, « La faculté de médecine », La Victoire de la démocratie, 25 novembre 1879.

7. Paul Bert, dans son rapport du 16 mars 1874 , soumis à l'assemblée nationale, sur la création des nouvelles facultés de médecine, avait évalué à plus de 800 le nombre des étudiants qui devaient fréquenter la faculté de Bordeaux.

8. Louis Liard arrive à Bordeaux comme chargé de cours à la faculté des lettres. Il est adjoint au maire, délégué à l'instruction publique de 1877 à 1880. De 1884 à 1902, il est directeur de l'enseignement supérieur au ministère de l'instruction publique et prépare les décrets Goblet de 1885 ainsi que la loi du 10 juillet 1896 qui crée une université par académie. "Pro Scientia Urbe et Patria : l'architecture de la faculté de médecine et de pharmacie de Bordeaux, 1876-1888 et 1902-1922 ", Revue archéologique de Bordeaux, op. cit., p. 138. 
9. Arch. mun. Bordeaux, A. Gambier, «La faculté de médecine », La Victoire de la démocratie, 25 novembre 1879.

10. Sur cette volonté de l'État de rationalité économique et non stylistique, voir Jean-Michel Leniaud, "Charles Garnier: un opposant à la centralisation stylistique de la commande publique ", article à paraître, p. 2. Je remercie Jean-Michel Leniaud de m'avoir communiqué cet article qui éclaire d'un jour nouveau cette problématique.

11. Arch. mun. Bordeaux, $6872 \mathrm{M} 24$, lettre du ministre de l'instruction publique et des beauxarts, Wallon, au vicomte de Pelleport Burete, maire de Bordeaux, le 22 avril 1875.

12. Ibid., extrait du registre des délibérations du conseil municipal de la ville de Bordeaux, séance du 4 avril 1876; "Concours pour la construction d'une faculté mixte de médecine et de pharmacie ", G.A.B., 31 mai 1876, $12^{\mathrm{e}}$ année, $\mathrm{n}^{\circ} 10$; Arch. mun. Bordeaux, 6872 M 27, « Programme du concours pour la construction d'une faculté mixte de médecine et de pharmacie à Bordeaux ». 13. Par calorifères.

14. Arch. mun. Bordeaux, 6872 M 27, «Programme du concours pour la construction d'une faculté mixte de médecine et de pharmacie à Bordeaux ", article 4.

15. «Les laboratoires d'anatomie, de physique, de chimie et de physiologie doivent avoir un accès facile avec le grand et le petit amphithéâtre de médecine et les laboratoires de pharmacie avec l'amphithéâtre de médecine ». Ibid., article 10.

16. Ibid., article 9.

17. Ibid., article 3.

18. L'exposition se tenait dans la salle de la Société des amis des arts, rue Vital Carles, à Bordeaux.

19. Arch. mun. Bordeaux, $6872 \mathrm{M} 24$, extrait du registre des délibérations du conseil municipal de la ville de Bordeaux, séance du 4 avril 1876, observations générales, article 4.

20. Projet $n^{\circ} 40$ : «Pro Scientia, Urbe et Patria ».

21. Seuls Ferdinand Dutert (1845-1906) et Mathurin-Eugène Calinaud (1843-1907) proposent un plan barlong ouvrant la façade au sud sur la rue Élie Gintrac.

22. Pour ne pas laisser une trouée d'aspect fâcheux sur la place d'Aquitaine ni trop resserrer contre les constructions voisines les services de l'anatomie, qui devaient être isolés, le jury préféra une implantation antérieure de l'édifice. On préservait aussi la possibilité d'une extension ultérieure pour les services de la faculté de pharmacie après acquisition des maisons sur la rue Leteyre.

23. «Les limites actuelles de mon plan pourront par suite être maintenues en les enfonçant simplement dans le trapèze qui représente notre terrain ", Arch. mun. Bordeaux, 6872 M 26, lettre de Pascal à Baysselance, 12 octobre 1879, puis «J'ai chargé M. Coëque Verdier (son inspecteur) de faire un tracé de l'emplacement occupé par le monument, dans le cas du recul de 10,00 m., obtenu par un glissement sur l'axe ». Ibid., lettre de Pascal, 10 novembre 1879.

24. La suppression du grand amphithéâtre entraîne une économie de 200000 francs. Elle est due à la réduction d'étendue de la faculté et principalement à la création d'une nouvelle faculté de médecine à Toulouse en 1878. Arch. mun. Bordeaux $6872 \mathrm{M} \mathrm{24}$, extrait du registre des délibérations du conseil municipal, 30 décembre 1879. On maintient dans les locaux de la caserne Raphaël les sections de pharmacie, physique, chimie et histoire naturelle. Pascal ne doit donc construire « qu'une » faculté de médecine, amputée de son grand amphithéâtre.

25. Arch. mun. Bordeaux, 6872 M 26, lettre de Pascal à Baysselance, 16 février 1879.

26. Le carrelage de mosaïque de marbre, d'une épaisseur de $4,5 \mathrm{~cm}$ est posé sur une couche de béton. Les cubes font $1,5 \mathrm{~cm}$ de côté.

27. Un voyage effectué en Angleterre et en Écosse au début des années quatre-vingt lui avait permis d'étudier l'architecture de l'École de médecine d'Édimbourg et des Universités de Glasgow et d'Aberdeen. Jean-Louis Pascal, « Facultés de médecine », R.G.A.T.P., 1884, p. 207-218 et 260-289. 
28. Ordre comparable pour son chapiteau à celui que Pascal utilise avec Coquart dans le monument à Henri Regnault. Claude Laroche, «Pro Scientia Urbe et Patria : l'architecture de la faculté de médecine et de pharmacie de Bordeaux, 1876-1888 et 1902-1922 », Revue archéologique de Bordeaux, op. cit., note 47 p. 161.

29. Ils conservent des collections africaines, océaniennes et asiatiques, réunies principalement par des médecins ayant travaillé outre-mer à la fin du XIX ${ }^{\mathrm{e}}$ siècle et au début du XX ${ }^{\mathrm{e}}$ siècle. Ibid., note 45, p. 155.

30. Arch. mun. Bordeaux, $6872 \mathrm{M} \mathrm{26}$, lettre de Pascal à Dormoy, adjoint au maire aux travaux publics, 10 octobre 1884 .

31. Plaquettes de $0,07 \mathrm{~cm}$ d'épaisseur, ce qui permet de remplacer avec beaucoup de facilité les marches cassées ou usées sans autre dépense que la fourniture de la matière première et sans occasionner de dégâts aux murs.

32. La maison Gauthier et Pozzi, 143 quai de Valmy, estimait la dépense à 27626,71 francs.

33. Arch. mun. Bordeaux, 6872 M 38, extrait du registre des délibérations du conseil municipal de la ville de Bordeaux, séance du 6 mai 1887.

34. Arch. mun. Bordeaux, 6872 M 38, lettre de Daumet, Paris, 13 juillet 1887, visée par Pascal. C'est cette maison qui sera choisie: Arch. mun. Bordeaux, $6872 \mathrm{M} \mathrm{38}$, adjudication, faculté de médecine, établissement d'escaliers mixtes en fer et pierre, 28 juillet 1887.

35. Arch. mun. Bordeaux, $6872 \mathrm{M} \mathrm{38}$, lettres de Pascal au maire, 29 janvier et 18 février 1887. Pascal impose un escalier secondaire en fer et pierre à cause de sa grande fréquentation. Quant aux escaliers menant aux combles (pavillons milieu des rues Broca et Gintrac), prévus en bois et pierre, Pascal n'aurait fait des économies qu'en les construisant tout en bois, ce à quoi il se refusait, ces escaliers étant les seuls à mener aux combles en cas d'incendie.

36. Ibid., ville de Bordeaux, construction de la faculté de médecine, ossature et rampes en fer des escaliers, mémoire général, 28 juillet 1887.

37. Sa longueur est de 3,50 m. Il a coûté 9163,25 fr. Arch. mun. Bordeaux, 6872 M 42, mairie de la ville de Bordeaux, construction de la faculté de médecine, décoration en carton-pâte, extrait du devis de Pascal, 10 nov. 1879.

38. Arch. mun. Bordeaux, 6872 M 27, lettre de Pascal à l'adjoint délégué aux travaux publics, $1^{\text {er }}$ août 1879 .

39. Arch. nat., $\mathrm{F}^{21} 4371$, lettre de Pascal au ministre des beaux-arts, 8 décembre 1884 .

40. Les statues devaient mesurer entre 2,90 et $3 \mathrm{~m}$, en y comprenant 10 à $15 \mathrm{~cm}$ d'un socle carré, réalisé par Pascal, ayant $0,90 \mathrm{~cm}$ de côté. Il était prévu un prix pour les deux statues de $24000 \mathrm{fr}$. L'État en payant la moitié, plus les marbres. Or les sculpteurs trouvaient la somme insuffisante. Le ministre se dit prêt à rajouter $3000 \mathrm{fr}$, si la ville acceptait de rajouter la même somme. Pascal devra réduire la hauteur des statues à $2 \mathrm{~m}$; Barrias et Cavelier acceptent alors la commande pour 15000 francs. Arch. nat., $\mathrm{F}^{21}$ 4371, dépêche du préfet au directeur des beaux-arts, 18 janvier 1889 .

41. Arch. nat., $\mathrm{F}^{21}$ 4371, lettre de Pascal au directeur des beaux-arts, 12 février 1888.

42. Ibid., lettre de Pascal au ministre des beaux-arts, 8 décembre 1884.

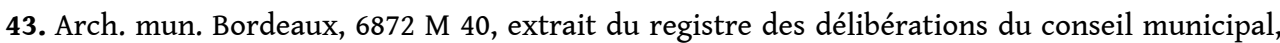
séance du 11 novembre 1887, rapporteur : Laroque.

44. Arch. nat., $\mathrm{F}^{21} 4371$, lettre de Pascal au directeur des beaux-arts, 2 septembre 1889.

45. Arch. mun. Bordeaux, 6872 M 47, « Deux statues », La Petite Gironde, 25 nov. 1893.

46. C'est l'entreprise Lamire frères qui les met en place pour 12000 francs. Arch. mun. Bordeaux, 6872 M 47, lettre au maire de Paul Mialhe, 10 nov. 1893.

47. Arch. nat., $\mathrm{F}^{21} 4371$, extrait du registre des délibérations du conseil municipal de la ville de Bordeaux, séance du 7 août 1888 .

48. Ces bustes devaient mesurer $1,05 \mathrm{~m}$. de hauteur, $0,63 \mathrm{~m}$ de largeur au socle sur $0,55 \mathrm{~m}$ de profondeur. Ibid., lettre de Pascal au ministre des beaux-arts, 8 décembre 1884. 
49. Botaniste français né à Lyon, il est à l'origine d'une méthode de classification des plantes, largement développée par son neveu Antoine-Laurent.

50. Breton né à Quimper, ayant fait ses études à Nantes et professeur à la faculté de médecine de Paris. Il fonda la médecine anatomoclinique.

51. Originaire de l'Ain, étudiant à Lyon, meurt médecin des hôpitaux de Paris à 31 ans. Il fonda l'anatomie générale et contribua au développement de l'histologie.

52. Chirurgien de Louis XVIII et de Charles X, il fut l'un des fondateurs de l'anatomie pathologique.

53. Né et mort à Paris, chimiste et financier célèbre.

54. On songea d'abord à Tournefort, botaniste né à Aix, ayant étudié à Montpellier, professeur à Paris, au Jardin des Plantes et au collège de France ; il fut remplacé par Bernard de Jussieu, le vrai fondateur de la méthode naturelle en botanique. Ambroise Paré, chirurgien barbier du XVI siècle né à Laval, fut remplacé par Dupuytren.

55. Arch. mun. Bordeaux, 6872 M 40, lettre du doyen au recteur, 23 mai 1885.

56. Arch. nat., $\mathrm{F}^{21} 4371$, lettre de Pascal au ministre des beaux-arts, 8 décembre 1884.

57. Il s'agit des sculpteurs Aury (pour le médaillon de Bouillaud), Chappuy (pour Parrot), Basset (pour Dufour), Beylard (pour Gratiolet), Bourgeois (pour Cruvheiler), Choppin (pour Broca), Chrétien (pour Gintrac), Faraill (pour Larrey), Granet (Magendie), Hexamer (Bert), Leclaire (Baudrimont), Leroux (Depaul), Mengin (Lesson), Prévot (Clémot), Arch. nat., $\mathrm{F}^{21} 4371$, note de la direction des beaux-arts. Les quatorze médaillons en marbre des Pyrénées sont payés quatorze mille francs, Arch. nat., $\mathrm{F}^{21} 4371$, lettre du 22 juin 1889 du préfet de la Gironde au ministre. Ils mesurent $0,86 \mathrm{~m}$ de diamètre et offrent une saillie de $0,15 \mathrm{~cm}$.

58. Arch. nat., $\mathrm{F}^{21} 4371$, lettre de Pascal au directeur des beaux-arts, 21 octobre 1887.

59. Arch. nat., $\mathrm{F}^{21} 4371$, Bordeaux, le 16 juin 1887, lettre du maire au ministre de l'instruction publique et des beaux-arts.

60. «L'État avait la libre disposition du travail ; cependant nous vous serions reconnaissants de vouloir bien confier l'exécution de quelques médaillons aux artistes Bordelais dont nous aurions l'honneur de vous soumettre les noms ». Ibid.

61. Arch. mun. Bordeaux, 6872 M 33, lettre de Pascal au maire, 21 août 1885.

62. Pascal fut obligé de mettre en concurrence cinq artistes dont deux Bordelais, Mora et Venturini, et trois Parisiens : Hamel, Germain, et Corbel. Hamel proposait le moindre prix (30 115 fr.) : «Un artiste éminent, chevalier de la légion d'honneur, membre de tous les jurys des arts décoratifs, à la tête d'une des maisons les mieux outillées qui soient, de plus cet artiste me propose de n'envoyer à Bordeaux que des artistes et contremaîtres pour diriger, tandis qu'il y embaucherait les ouvriers sculpteurs, il me semble que les intérêts qui préoccupaient M. Dormoy sont suffisamment sauvegardés ». Ibid.

63. Arch. mun. Bordeaux, $6872 \mathrm{M} \mathrm{26}$, lettre de Pascal à Dormoy, adjoint au maire aux travaux publics, 10 octobre 1884 .

64. Arch. nat., $\mathrm{F}^{21} 4371$, lettre de Pascal au ministre des beaux-arts, 8 décembre 1884.

65. La liste comprenait les noms de Granet, Loiseau, Michel, Massoulle, Hugoulin, Gauthier, Vital Dubray, Chrétien, Béguine, Lefebvre. Prévot ne faisait pas partie de la liste, il fut imposé par Louis Liard. Arch. nat., $\mathrm{F}^{21}$ 4371, lettre du ministre à Pascal, 5 mars 1885.

66. Arch. mun. Bordeaux, $6872 \mathrm{M} \mathrm{40,} \mathrm{lettre} \mathrm{de} \mathrm{Pascal} \mathrm{au} \mathrm{maire,} 9$ juin 1885.

67. Il s'agit de deux statues à l'antique, une femme tenant un caducée et un homme tenant une couronne de laurier, qui ornent deux niches de part et d'autre de l'entrée sous le portique de l'hôtel de ville. Elles sont signées de Prévot et datées de 1869.

68. Arch. mun. Bordeaux, 6872 M 40, lettre de Pascal au maire, 9 juin 1885.

69. Au rez-de-chaussée, le corps de bâtiment en façade ménage un vestibule qui donne accès à gauche au grand escalier sous lequel on doit passer pour atteindre la salle des actes. 
70. Elle mesure $2,78 \mathrm{~m}$ de large et $4,17 \mathrm{~m}$ de haut.

71. Le tout pour 5700 fr. par Jouffre, 110 rue Sainte Catherine (en fait 3990,00 fr. après rabais). Arch. mun. Bordeaux, $6872 \mathrm{M} 44$, extrait du registre des délibérations du conseil municipal de la ville de Bordeaux, séance du 30 nov. 1888.

72. La table a un développement de $10 \mathrm{~m}$ sur 1,30 m de large. Elle a coûté 1800 fr., les fauteuils, $1200 \mathrm{fr}$., le bureau avec estrade, $800 \mathrm{fr}$., soit un total de $3800 \mathrm{fr}$.

73. Arch. mun. Bordeaux, $6872 \mathrm{M} 53$, devis du mobilier de la salle des actes et cabinet du doyen, Pascal et Mialhe, 26 novembre 1893.

74. Le tableau est retiré du Dépôt ( $n^{\circ}$ inventaire 1242) le 5 mai 1890 pour être expédié à la faculté de médecine. Arch. nat., $\mathrm{F}^{21}$ 4371, note du bureau des beaux-arts, 15 mai 1890.

75. Ibid., lettre du ministre de l'instruction publique et des beaux-arts au préfet, 18 juillet 1888 .

76. Á hauteur de 5000 francs. Ibid., Lettre du ministre au préfet, 10 novembre 1888.

77. «M. Dantan a été chargé de reproduire pour ma faculté de médecine la cérémonie de remise de cet édifice à l'État, j'ai été heureux que le hasard de ma présence à Bordeaux [J.-L. Pascal fait partie du jury du concours pour le monument aux Girondins] m'ait permis de m'entendre avec lui pour choisir le meilleur emplacement pour son œuvre et pour la décoration d'une des salles les plus importantes de l'établissement. J'ignorais la faveur accordée à notre monument ». Arch. nat., $\mathrm{F}^{21} 4371$, lettre de Pascal au directeur des beaux-arts, 12 août 1889.

78. Arch. mun. Bordeaux $6872 \mathrm{M} \mathrm{48,} \mathrm{lettre} \mathrm{de} \mathrm{Pascal} \mathrm{au} \mathrm{maire,} 28$ novembre 1889. Une lettre du maire de Bordeaux Baysselance au ministre le destine au grand amphithéâtre. Ibid., lettre du maire au ministre, 2 décembre 1889. Elle fut définitivement attribuée à la salle des actes puisqu'un devis fait état d'une «modification à apporter au lambris pour baisser le tableau de M. Dantan et refaire par la suite la frise de la corniche ». Arch. mun. Bordeaux, 6872 M 53, devis $\mathrm{du}$ mobilier de la salle des actes et cabinet du doyen, Pascal et Mialhe, 26 novembre 1893. Il décore actuellement la partie gauche de la galerie qui double le vestibule (mur mitoyen avec l'amphithéâtre Gintrac).

79. Arch. mun. Bordeaux, 6872 M 48, lettre de Pascal au maire, 23 novembre 1888.

80. Fixées sur châssis. Hauteur : 3, 19 m. Largeur : 1, 73 m. Arch. mun. Bordeaux, 6872 M 48, lettre du directeur des beaux-arts Henri Roujon au maire, 30 mars 1892.

81. Les frais des modèles étaient de 6000 francs.

82. «Monsieur Galland est assurément un des premiers artistes de cette spécialité décorative, en ce qu'il est aussi habile à manier la figure que la flore, les motifs décoratifs, l'architecture, tout ce qui doit se fondre dans un travail de ce genre. Il est le professeur de décoration à notre École des beaux-arts ; il est le directeur des travaux aux Gobelins. C'est le dessus du panier. » Arch. mun. Bordeaux, 6872 M 48 : lettre de Pascal au maire, 24 décembre 1889.

83. Arch. mun. Bordeaux, 6872 M 57, extrait du registre des délibérations du conseil municipal, séance du 8 mai 1903.

\section{RÉSUMÉS}

Lorsque Jean-Louis Pascal remporte le concours pour la construction de la faculté de médecine et de pharmacie de Bordeaux, c'est déjà un architecte reconnu, qui vient d'être nommé architecte de la Bibliothèque nationale à Paris. Il réalise à Bordeaux un monument au plan typiquement «Beaux-arts» mais un bâtiment rationnel, qui tient compte des nouvelles découvertes de la 
médecine et intègre les matériaux modernes du XIX ${ }^{\mathrm{e}}$ siècle. L'austérité du bâtiment est aussi due aux contraintes budgétaires qui s'imposèrent à l'architecte, l'obligeant même à remanier son plan, amputé du grand amphithéâtre que celui-ci souhaitait pour une grande faculté. Son extension destinée à la faculté de pharmacie, réalisée au début du $\mathrm{XX}^{\mathrm{e}}$ siècle après la première guerre mondiale, proposera une façade légèrement surannée, achevée après la mort de JeanLouis Pascal.

When Jean-Louis Pascal wins the competition to design the Faculty of Medicine and Pharmacy in Bordeaux, he is already a well-known architect who has just been appointed as architect charged with the Bibliothèque nationale in Paris. In Bordeaux, he designs a monument with a typically Beaux-arts plan, yet a rational building which takes into account some new discoveries in the field of medicine and uses $19^{\text {th }}$ century modern materials. The severity of the building is partly due to financial difficulties the architect had to come up against. He was even compelled to rework his plan and to cut out the great amphitheatre he considered desirable in any major faculty. The enlargement allocated to the Faculty of Pharmacy and built in the early $20^{\text {th }}$ century, after the First World War, will show a slightly old-fashioned façade, completed after Jean-Louis Pascal's death.

Als Jean-Louis Pascal den Wettbewerb für den Bau der Fakultät für Medizin und Pharmazie von Bordeaux gewann, hatte er schon einen guten Ruf erworben und war gerade zum Architekten der Pariser Nationalbibliothek ernannt worden. Er errichtete in Bordeaux ein Bauwerk im typischen Beaux-arts- Stil, dabei jedoch nach rationellen Kriterien, um den neuen medizinischen Errungenschaften Rechnung zu tragen unter Verwendung der modernen Materialien des 19. Jahrhunderts. An dem nüchternen Charakter des Gebäudes lassen sich die finanziellen Bedingungen erkennen, die dem Architekten vorgeschrieben wurden. Deswegen musste er den großen geplanten Hörsaal aufgeben, den er bei einem solchen Universitätsbau eigentlich für notwendig hielt. Der Erweiterungsbau, der Fakultät für Pharmazie gewidmet, entstand im frühen 20.Jahrhundert erst nach dem ersten Weltkrieg. Die Fassade im etwas veralteten Stil wurde nach dem Tode Jean-Louis Pascals beendet.

\section{AUTEUR}

\section{ANNE RICHARD-BAZIRE}

Anne Richard-Bazire, née en 1961, diplômée du second cycle de l'École du Louvre, a réalisé son mémoire de DEA à l'École pratique des hautes études sur « Jean-Louis Pascal et la salle des périodiques de la Bibliothèque nationale ». Actuellement doctorante à l'École pratique des hautes études, elle prépare sous la direction de Jean-Michel Leniaud une thèse sur « Jean-Louis Pascal, architecte ». Elle est l'auteur de « Jean-Louis Pascal et la création de la salle des périodiques à la Bibliothèque nationale, 1883-1936", dans LHA, 2001, $\mathrm{n}^{\circ}$ 1, de " Un siècle de réflexion sur la construction des bibliothèques", dans Des palais pour des livres. Labrouste, Sainte-Geneviève et les bibliothèques, sous la direction de Jean-Michel Leniaud, Paris, Maisonneuve et Larose, 2002. Elle a participé à la rédaction des catalogues d'expositions Les bibliothèques parisiennes, architecture et décor, Paris, AAVP, 2002, et Autour de Notre-Dame, Paris, AAVP, 2003. Elle est l'auteur de « Le ministère de l'agriculture de la rue de Varenne par Emmanuel Brune, l'alliance de la science et de l'art ", dans LHA, 2004, nº 8. Adresse électronique : annerichardbazire@hotmail.com 